\title{
Effects of dietary and physical activity interventions on the risk of type 2 diabetes in South Asians: meta-analysis of individual participant data from randomised controlled trials
}

\author{
Anne Karen Jenum ${ }^{1} \cdot$ Idunn Brekke ${ }^{2,3} \cdot$ Ibrahimu Mdala $^{1} \cdot$ Mirthe Muilwijk $^{4}$ - Ambady Ramachandran ${ }^{5,6}$. \\ Marte Kjøllesdal $^{7} \cdot$ Eivind Andersen $^{8} \cdot$ Kăre R. Richardsen $^{9} \cdot$ Anne Douglas $^{10} \cdot$ Genevieve Cezard $^{10,11} \cdot$ Aziz Sheikh $^{10}$. $^{2}$ \\ Carlos A. Celis-Morales ${ }^{12}$ • Jason M. R. Gill ${ }^{12}$ - Naveed Sattar ${ }^{12}$ • Raj S. Bhopal ${ }^{10}$ • Erik Beune . Karien Stronks $^{4}$. \\ Per Olav Vandvik ${ }^{13}$ • Irene G. M. van Valkengoed ${ }^{4}$
}

Received: 20 December 2018 / Accepted: 15 April 2019 / Published online: 15 June 2019

(C) Springer-Verlag GmbH Germany, part of Springer Nature 2019

\begin{abstract}
Aims/hypothesis Individuals of South Asian origin have a high risk of type 2 diabetes and of dying from a diabetes-attributable cause. Lifestyle modification intervention trials to prevent type 2 diabetes in high-risk South Asian adults have suggested more modest effects than in European-origin populations. The strength of the evidence of individual studies is limited, however. We performed an individual participant data meta-analysis of available RCTs to assess the effectiveness of lifestyle modification in South Asian populations worldwide.

Methods We searched PubMed, EMBASE, Cochrane Library and Web of Science (to 24 September 2018) for RCTs on lifestyle modification interventions incorporating diet and/or physical activity in South Asian adults. Reviewers identified eligible studies and assessed the quality of the evidence. We obtained individual participant data on 1816 participants from all six eligible trials (four from Europe and two from India). We generated HR estimates for incident diabetes (primary outcome) and mean differences for fasting glucose, $2 \mathrm{~h}$ glucose, weight and waist circumference (secondary outcomes) using mixed-effect meta-analysis overall and by pre-specified subgroups. We used the Grading of Recommendations, Assessment, Development and Evaluation (GRADE) system to rate the quality of evidence of the estimates. The study is registered with the International Prospective Register of Systematic Reviews ([PROSPERO] CRD42017078003).

Results Incident diabetes was observed in $12.6 \%$ of participants in the intervention groups and in $20.0 \%$ of participants in the control groups. The pooled HR for diabetes incidence was $0.65\left(95 \%\right.$ CI $\left.0.51,0.81 ; I^{2}=0 \%\right)$ in intervention compared with
\end{abstract}

Electronic supplementary material The online version of this article (https://doi.org/10.1007/s00125-019-4905-2) contains peer-reviewed but unedited supplementary material, which is available to authorised users.

Anne Karen Jenum

a.k.jenum@medisin.uio.no

1 General Practice Research Unit (AFE), Department of General Practice, Institute of Health and Society, Faculty of Medicine, University of Oslo, Post Box 1130 Blindern, 0318 Oslo, Norway

2 Centre for Welfare and Labour Research, Norwegian Social Research, OsloMet - Oslo Metropolitan University, Oslo, Norway

3 Faculty of Health Sciences, Department of Nursing and Health Promotion, OsloMet - Oslo Metropolitan University, Oslo, Norway

4 Department of Public Health, Amsterdam Public Health Research Institute, University of Amsterdam, Amsterdam, the Netherlands

5 India Diabetes Research Foundation, Chennai, India

6 Dr. A. Ramachandran's Diabetes Hospitals, Chennai, India
7 Department of Community Medicine and Global Health, Institute of Health and Society, Faculty of Medicine, University of Oslo, Oslo, Norway

8 Faculty of Humanities, Sports and Educational Science, University of South-Eastern Norway, Borre, Norway

9 Department of Physiotherapy, Faculty of Health Sciences, OsloMet Oslo Metropolitan University, Oslo, Norway

10 Usher Institute of Population Health Sciences and Informatics, University of Edinburgh, Edinburgh, UK

11 Population and Health Research Group, School of Geography and Sustainable Development, University of St Andrews, Fife, Scotland, UK

12 Institute of Cardiovascular and Medical Sciences, University of Glasgow, Glasgow, UK

13 Department of Medicine, Innlandet Hospital Trust, Gjøvik, Norway 


\section{Research in context}

\section{What is already known about this subject?}

- Individuals of South Asian origin have a substantially higher risk of type 2 diabetes (referred to as diabetes hereafter) and a higher proportion of deaths attributable to diabetes than individuals of European origin

- Lifestyle modification has been found to be effective in preventing diabetes in European-origin populations

- Diabetes prevention lifestyle modification trials among South Asian adults seem to be less effective than in other populations

\section{What is the key question?}

- Do lifestyle modification interventions prevent diabetes in South Asian adults?

\section{What are the new findings?}

- This systematic review and individual participant data meta-analysis changes our thinking on the assumed limited effect of lifestyle modification interventions in high-risk South Asian individuals; we found a meaningful $35 \%$ relative reduction and a $7.4 \%$ absolute reduction in diabetes incidence among people of South Asian origin receiving lifestyle modification interventions, compared with control groups

- Effects on diabetes incidence were consistent for all a priori pre-specified subgroups

- Modest effects were found for $2 \mathrm{~h}$ glucose, weight and waist circumference, consistent across all pre-specified subgroups, except for weight, which differed by region

\section{How might this impact on clinical practice in the foreseeable future?}

- This meta-analysis strongly supports the value of lifestyle modification interventions as part of a strategy to prevent diabetes in individuals of South Asian origin; this is important given that the burden of diabetes is substantial and growing, particularly in this group

control groups. The absolute risk reduction was 7.4\% (95\% CI 4.0, 10.2), with no interactions for the pre-specified subgroups (sex, BMI, age, study duration and region where studies were performed). The quality of evidence was rated as moderate. Mean difference for lifestyle modification vs control groups for $2 \mathrm{~h}$ glucose was $-0.34 \mathrm{mmol} / \mathrm{l}\left(95 \% \mathrm{CI}-0.62,-0.07 ; I^{2}=50 \%\right)$; for weight $-0.75 \mathrm{~kg}\left(95 \% \mathrm{CI}-1.34,-0.17 ; I^{2}=71 \%\right)$ and for waist $-1.16 \mathrm{~cm}\left(95 \% \mathrm{CI}-2.16,-0.16 ; I^{2}=75 \%\right)$. No effect was found for fasting glucose. Findings were similar across subgroups, except for weight for European vs Indian studies $(-1.10 \mathrm{~kg}$ vs $-0.08 \mathrm{~kg}, p=0.02$ for interaction).

Conclusions/interpretation Despite modest changes for adiposity, lifestyle modification interventions in high-risk South Asian populations resulted in a clinically important $35 \%$ relative reduction in diabetes incidence, consistent across subgroups. If implemented on a large scale, lifestyle modification interventions in high-risk South Asian populations in Europe would reduce the incidence of diabetes in these populations.

Keywords Diet · Individual participant data meta-analysis $\cdot$ Lifestyle intervention $\cdot$ Physical activity $\cdot$ Prevention $\cdot$ RCT $\cdot$ South Asians · Type 2 diabetes

\section{Abbreviations \\ AMC \\ DHIAAN}

GRADE

NNT

PODOSA
Academic Medical Center

Diabetes Intervention Study in Hindustani

Surinamese

Grading of Recommendations, Assessment, Development and Evaluation

Number needed to treat

Prevention of Diabetes and Obesity in South Asians
PROSPERO International Prospective Register of Systematic Reviews

\section{Introduction}

Type 2 diabetes (hereafter referred to as diabetes) and its complications constitute a major threat to global health. Populations of South Asian origin have at least a two to four 
times higher risk than European-origin populations [1, 2]; they also develop diabetes and its complications at a younger age $[3,4]$. Patterns of fat deposition, low lean mass [5] and low birthweight [6] are considered to contribute to early-onset insulin resistance and diabetes in South Asians, exacerbated by changing lifestyle, urbanisation and migration. Further, the proportion of deaths attributable to diabetes is almost $50 \%$ higher in South Asians than in populations of European origin, with no clear signs that the risk will level off over time [7]. Therefore, adequate actions for prevention of diabetes among South Asians are imperative.

From 2001, efficacy trials have documented a reduction in diabetes incidence in high-risk adults of up to $58 \%$ through lifestyle modification interventions incorporating a healthy diet and/or physical activity [8-12]. Recent meta-analyses of efficacy and pragmatic diabetes prevention trials found a $30-40 \%$ reduction in diabetes incidence $[13,14]$. However, none of these meta-analyses reported effects specifically for South Asians [13, 14], although several lifestyle intervention trials, culturally adapted to suit the specific needs, cultural norms and values of the South Asian populations in different contexts, have been developed and tested [15-18]. Some trials in South Asian populations appeared moderately successful, but the effectiveness differed across studies. Although a few previous reviews have explored the effects of lifestyle modification interventions on glucose measures and anthropometric outcomes in South Asians [19], many were narrative and limited by inconsistent outcome reporting, and none reported estimates for diabetes incidence [20]. Owing to small numbers in individual studies and low power - the a priori estimation of effects was too optimistic, drop out was higher than anticipated or the incidence of outcomes was lower than expected - the strength of the evidence of individual studies is limited and further exploration of effects (e.g. subgroup analyses) are hampered.

Given the current knowledge gap about the effectiveness of lifestyle modification interventions in this high-risk population, we aimed to assess the overall effect from RCTs with lifestyle interventions involving dietary modification and/or physical activity to prevent diabetes in adult South Asians, using individual participant data meta-analysis [21]. In addition, we explored whether the effects differed by sex, BMI, age, study duration or study region.

\section{Methods}

The plan for this individual participant data meta-analysis was registered with the International Prospective Register of Systematic Reviews ([PROSPERO] registration CRD42017078003). The reporting is in line with the Preferred Reporting Items for Systematic Reviews and
Meta-Analysis of Individual Participant Data (PRISM-P) [22].

Eligibility criteria Individual and cluster RCTs that compared the effect of lifestyle modification interventions incorporating a healthy diet and/or physical activity with usual care/control groups not receiving the lifestyle intervention to prevent diabetes in the South Asian adult population ( $\geq 18$ years) worldwide were eligible. To reduce the risk of bias in the estimates, studies were included only if judged to be at least of moderate quality by the Quality Assessment Tool for Quantitative Studies (see below) [23]. We decided a priori to exclude studies exclusively on drugs and surgical interventions, alone or in combination with lifestyle modification, and studies evaluating physical activity components not consisting of cardiovascular exercise (e.g. yoga, resistance training) and single dietary components (such as vitamin D supplementation).

Search strategy We searched PubMed, EMBASE, Cochrane Library and Web of Science, first from the start to 30 September 2017 to allow for inclusion of studies for the individual participant data meta-analysis (search strategy shown in electronic supplementary material [ESM] Methods), supplemented with reference list tracing of key reviews $[19,20$, $24,25]$, included studies $[12,15-18,26]$ and by searching trial registers [27]. The search was updated on 24 September 2018. The work builds on and complements our previous narrative systematic review of dietary and physical activity components recommended in experimental, quasi-experimental and before/after studies to prevent diabetes in South Asians [27].

Study selection and risk of bias assessment Two reviewers independently screened titles and abstracts for eligibility, thereafter full texts. The risk of bias was assessed at the study level using the Quality Assessment Tool for Quantitative Studies [23] by three reviewers, as we perceived the quality assessment tool to be vulnerable to differences in interpretation. For all processes, a discussion was held to reach consensus in cases of discrepancy. Only studies judged to be at least of moderate quality were included [23].

Data extraction Information on study design and population characteristics was extracted from published manuscripts, study protocols and personal communication with authors, using a piloted extraction form, by two reviewers working independently [27]. A discussion was held in case of discrepancies and a third independent reviewer arbitrated, if necessary. Principal investigators of eligible studies published prior to 30 September 2017 were contacted for permission to include individual participant data on sex, age, anthropometric measures and fasting and $2 \mathrm{~h}$ glucose results from oral glucose tolerance tests. To allow for transformation and analysis, the deadline for data inclusion was set to 1 March 2018. After 
each primary study gave ethical approval for the current study, data transfer agreements were signed before we received anonymised individual level data.

Data analysis and quality of the evidence The primary outcome was diabetes incidence, defined as fasting glucose $\geq 7.0 \mathrm{mmol} / \mathrm{l}$ and $/$ or $2 \mathrm{~h}$ glucose $\geq 11.1 \mathrm{mmol} / 1$ or registered as doctor diagnosed by self-report at follow-up visits $[12,16$, 26]. Secondary intermediate outcomes were changes in fasting glucose, $2 \mathrm{~h}$ glucose, body weight $(\mathrm{kg})$ and waist circumference $(\mathrm{cm})$. Except for one study with age stratified into nine groups [16], we used age in years. Analyses were based on an intention-to-treat approach. All principal investigators confirmed preliminary analyses after variable standardisation and data cleaning.

Cox proportional hazards models were used to estimate age-adjusted HRs for diabetes incidence. The three studies with men and women were also adjusted for sex. The proportional hazard assumption was tested separately for each covariate and also overall (global test) and found to be met. Time to diabetes was used as the dependent variable in our survival analysis, and was calculated from the trial start date to either the diabetes diagnosis or the end of each trial. For secondary outcomes, we determined mean differences with 95\% CIs, adjusted for baseline values for the outcome variable of interest, and age and sex when relevant, using the last available estimate [21]. Individual participant data meta-analyses were done in two stages. First, mixed regression models with random effects at individual level and group allocation as fixed effect were performed for each study. For secondary outcomes, we used generalised linear models with identity link function for studies with only one follow-up visit [17, 18]. Second, estimates from the multilevel models were used to estimate weighted averages across studies. We used the $I^{2}$ statistic to express the amount of variance attributable to study heterogeneity. A priori-defined subgroup analyses were performed for sex, baseline BMI $\geq 27.5 \mathrm{~kg} / \mathrm{m}^{2}$ or $<27.5 \mathrm{~kg} / \mathrm{m}^{2}$ [28], age $>44$ years or $<44$ years, short- (5-7 months) and long-term (2-3 years) study duration and region, testing for interactions between intervention and subgroups. We used Stata version 15.1 (StataCorp, College Station, TX, USA) for the regression analyses for each study and to generate weighted estimates across studies, and the metafor package in R (http://www.jstatsoft.org/v36/i03/) for the forest plots. Because of loss to follow-up in some studies, we performed a sensitivity analysis, applying within-trial multiple imputations [29]. In trials with multiple follow-up visits [15, 16, 26] we used REALCOM-IMPUTE [30] to account for correlation between repeated measures. In trials with one follow-up [17, 18], we used multiple imputation by chained equations [31]. For each trial, we created 20 imputed datasets, thereafter combined for pooled estimates. We also performed funnel plots to visually explore possible publication bias/small study effect.
Finally, we used the Grading of Recommendations, Assessment, Development and Evaluation (GRADE) approach to rate the quality of evidence and to generate absolute effect estimates for the outcomes [32]. In our previous systematic review, we identified RCTs that ended before or in 2015 and had not published results or updated their registrations [27]. Of the six trials that were identified and might have been eligible for the individual participant data meta-analysis, three have not yet reported, one has since published results [33] and two are ongoing. Although it is unclear if the underreporting is related to unsuccessful inclusion or negative results, it is indicative of a probable publication bias.

\section{Results}

Included studies We identified 4240 publications and, after removal of duplicates, 3009 titles and abstracts were screened, of which 2983 were excluded (ESM Fig. 1). Following fulltext review of 17 articles, six RCTs fulfilled the inclusion criteria for the individual participant data meta-analysis [12, 15-18, 26]. The characteristics of excluded studies [33-43] are given in ESM Table 1. We obtained individual participant data for all six eligible studies for the meta-analysis (Table 1). When cleaning the data for analyses, we identified 29 individuals from two studies with glucose levels indicative of diabetes at inclusion $[17,18]$. These individuals were excluded, leaving 1816 participants, of whom 604 (33\%) were women. Three studies included both sexes, two were in men $[18,26]$ and one was in women only [17]. Four studies were of 23 years' duration $[12,15,16,26]$, while two lasted 5 7 months $[17,18]$. Four studies were from Europe [15-18] and two from India [12,26]. Five studies combined a dietary and physical activity intervention $[12,15-17,26]$, while one used physical activity only (primarily floorball/field hockey) [18]. Individual and/or group-based educational sessions were provided with varying intensity, one supported by mobile phone messages [26]. One study offered home visits from a dietitian and involvement of family members [16], three were performed in the community $[15,17,18]$ and the two Indian studies were performed in a workplace setting $[12,26]$. The quality of five studies was rated as strong (ESM Table 2).

Loss to follow-up differed from more than $20 \%$ in two studies of 7 months' and 2 years' duration $[15,17]$ to 0 $2.3 \%$ in other studies that had 3 years' duration $[12,16]$ (ESM Table 3). No clear patterns of differences in baseline characteristics between the intervention and control groups were observed (ESM Table 4). The age range at inclusion was $18-80$ years, with mean age differing from 37 [18] to 52 years [16]. The mean BMI differed from $26 \mathrm{~kg} / \mathrm{m}^{2}$ in the Indian studies $[12,26]$ to $30.6 \mathrm{~kg} / \mathrm{m}^{2}$ in one European study [16]. At baseline, mean $2 \mathrm{~h}$ glucose levels were highest in the 


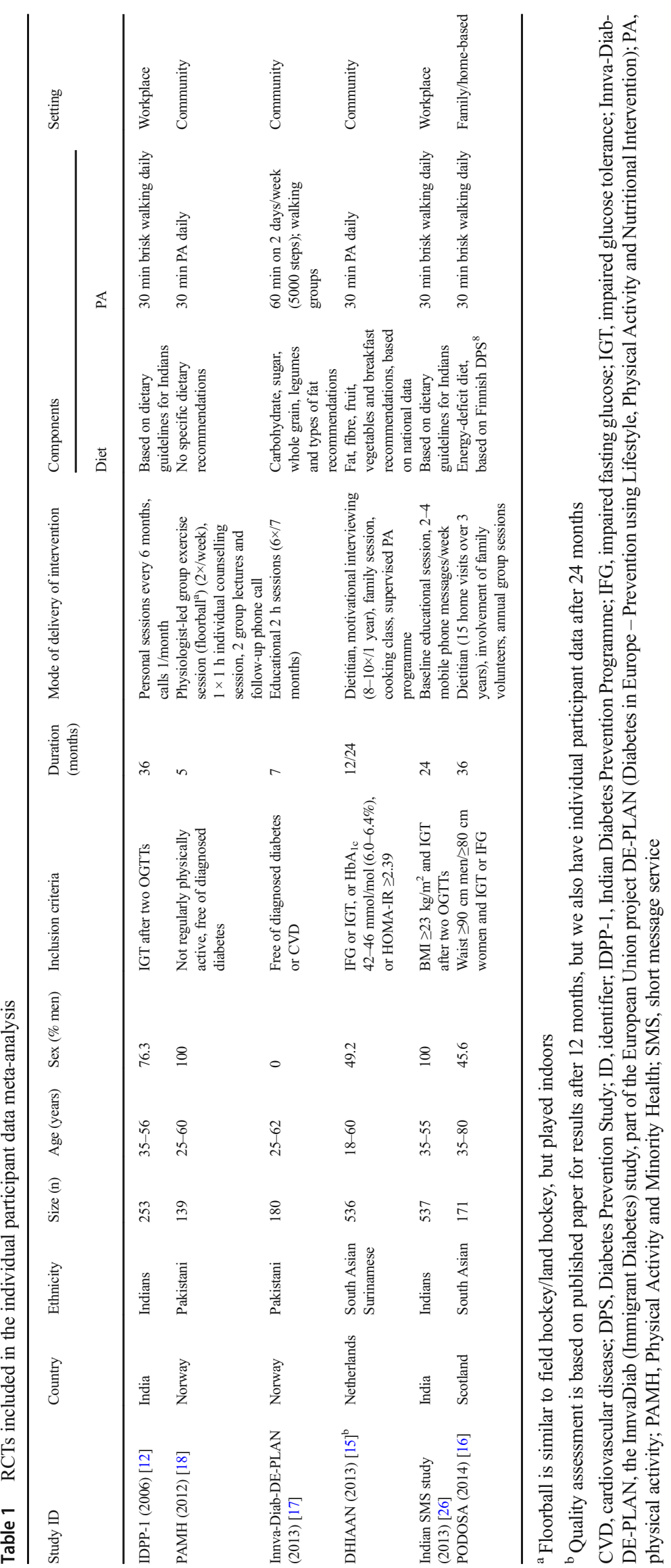


Table 2 Diabetes risks overall and within pre-specified subgroups

\begin{tabular}{|c|c|c|c|c|c|c|c|c|}
\hline Outcome & No. of studies & All cases $/ \mathrm{N}$ & Intervention $\mathrm{n} / \mathrm{N}$ & Control $\mathrm{n} / \mathrm{N}$ & $\mathrm{HR}^{\mathrm{a}}(95 \% \mathrm{CI})$ & $p$ value $e^{b}$ & $I^{2}$ & $\begin{array}{l}p \text { value } \\
\text { interaction }^{\mathrm{c}}\end{array}$ \\
\hline Overall & 6 & $294 / 1816$ & $118 / 936$ & $176 / 880$ & $0.65(0.51,0.81)$ & 0.0002 & 0 & \\
\hline \multicolumn{9}{|l|}{$\operatorname{Sex}^{\mathrm{d}}$} \\
\hline Men & 5 & $244 / 1211$ & $102 / 624$ & $142 / 587$ & $0.68(0.53,0.88)$ & 0.003 & 0 & 0.30 \\
\hline Women & 4 & $50 / 604$ & $16 / 312$ & $34 / 292$ & $0.48(0.26,0.88)$ & 0.02 & 0 & \\
\hline \multicolumn{9}{|l|}{ BMI, kg/m² } \\
\hline$\geq 27.5$ & 6 & $97 / 729$ & $38 / 362$ & $59 / 367$ & $0.64(0.34,1.20)$ & 0.15 & 46 & 0.99 \\
\hline$<27.5$ & 6 & $197 / 1087$ & $80 / 574$ & $117 / 513$ & $0.64(0.46,0.88)$ & 0.002 & 10 & \\
\hline \multicolumn{9}{|l|}{ Age, years } \\
\hline$>44$ & 6 & $170 / 946$ & $70 / 481$ & $100 / 465$ & $0.69(0.50,0.93)$ & 0.02 & 0 & 0.55 \\
\hline$\leq 44$ & 6 & $124 / 870$ & $48 / 455$ & $76 / 415$ & $0.59(0.41,0.85)$ & 0.005 & 0 & \\
\hline \multicolumn{9}{|l|}{ Study duration } \\
\hline 5-7 months & 2 & $4 / 319$ & $2 / 177$ & $2 / 142$ & $0.72(0.10,5.11)$ & 0.74 & 0 & 0.92 \\
\hline $2-3$ years & 4 & $290 / 1497$ & $116 / 759$ & $174 / 738$ & $0.64(0.51,0.81)$ & 0.0003 & 0 & \\
\hline \multicolumn{9}{|l|}{ Study region } \\
\hline Europe & 4 & $50 / 1026$ & $21 / 545$ & $29 / 481$ & $0.69(0.39,1.21)$ & 0.19 & 0 & 0.81 \\
\hline India & 2 & $244 / 790$ & $97 / 391$ & $147 / 399$ & $0.64(0.49,0.82)$ & 0.0006 & 0 & \\
\hline
\end{tabular}

${ }^{\mathrm{a}}$ HRs adjusted for age and sex

${ }^{\mathrm{b}} p$ values for HRs

${ }^{\mathrm{c}} p$ values for interaction term for subgroup analyses

${ }^{\mathrm{d}}$ One person in the control group had missing information about sex

No., number

Indian studies, which only included participants with persistently impaired glucose tolerance $[12,26]$.

Diabetes incidence Incident diabetes was observed in 118 of 936 participants $(12.6 \%)$ in the intervention group and in 176 of 880 participants $(20.0 \%)$ in the control group (adjusted HR 0.65 (95\% CI 0.51, 0.81; $p=0.0002, I^{2}=0 \%$ ) (Table 2 and Fig. 1). This reflects an absolute reduction of $7.4 \%(95 \% \mathrm{CI}$ $4.0,10.2)$ and a number needed to treat (NNT) of $14(95 \%$ CI $10.0,28.0)$. In subgroup analyses, we did not find differences in relative estimates of effects by sex, age, BMI, study duration or region. For the long-term studies, the estimates were identical after exclusion of one study with high loss to followup [15] (0.65 [95\% CI 0.51, 0.83], $p=0.0004)$. Overall, and for subgroups, we found no $\left(I^{2}=0 \%\right)$ or low between-study heterogeneity. According to GRADE, the quality of evidence can be rated as moderate; quality was rated down owing to some, but overall limited, concerns of risk of bias (e.g. lacking blinding of participants and providers) and possible publication bias, although not confirmed by visual inspection of funnel plots (data not shown).

Other outcomes For secondary outcomes, we observed a reduction in $2 \mathrm{~h}$ glucose $(-0.34 \mathrm{mmol} / 1$ [95\% CI -0.62 to $\left.-0.07], p=0.02, I^{2}=50 \%\right)$, weight $(-0.75 \mathrm{~kg}[95 \% \mathrm{CI}$ -1.34 to -0.17$], p=0.01, I^{2}=71 \%$ ) and waist circumference $\left(-1.16 \mathrm{~cm}[95 \% \mathrm{CI}-2.16\right.$ to -0.16$\left.], p=0.02, I^{2}=75 \%\right)$ in the intervention vs control group (Table 3 and ESM Fig. 2), with no effect for fasting glucose $(-0.03$ [95\% CI -0.10 to 0.04 , $p=0.37, I^{2}=0 \%$ ). No consistent subgroup differences in effects were found for secondary outcomes (ESM Table 5), although a difference in effect for weight $(-1.10 \mathrm{~kg} \mathrm{vs}-0.08 \mathrm{~kg}$, $p=0.02$ for interaction) was revealed for European vs Indian studies, with a similar but non-significant difference in the effect estimate for waist circumference $(-1.59 \mathrm{~cm}$ vs $-0.26 \mathrm{~cm}, p=0.09$ for interaction). The GRADE quality of evidence for $2 \mathrm{~h}$ glucose was rated as moderate, rated down owing to some concerns about risk of bias. For the other secondary outcomes, the quality was considered low because of imprecision and/or heterogeneity between studies (ESM Table 5). Sensitivity analyses after multiple imputations yielded similar effect estimates as the main analysis for secondary outcomes, although CIs were slightly wider and the result for weight was only borderline significant $(p=0.06$; results not shown). Last, the distribution curves for fasting and $2 \mathrm{~h}$ glucose at the last visit for all studies merged showed a larger shift to the right in the control than in the intervention group (ESM Fig. 3). In the intervention group, $4.5 \%$ had developed diabetes at the last follow-up based on fasting glucose values $\geq 7.0 \mathrm{mmol} / 1$ and $8.2 \%$ based on $2 \mathrm{~h}$ glucose values $\geq 11.1 \mathrm{mmol} / 1$, compared with $7.5 \%(p=0.017)$ and $14.9 \%$ $(p<0.0001)$, respectively, in the control group. 


\section{Discussion}

In this individual participant data meta-analysis we found that the overall incidence of diabetes was reduced by $35 \%$ in the group receiving the lifestyle modification intervention compared with the control group, with an absolute risk reduction of $7.4 \%$ and an NNT of $\sim 14$ over a mean of 2 years. Results for diabetes were consistent for men and women and for other key subgroups. The studies included participants with normal and impaired glucose regulation and a range of age and BMI values at baseline, indicating high external validity. We also found evidence of a modest effect on $2 \mathrm{~h}$ glucose and simple measures of adiposity (weight and waist circumference). Of note, however, at the last follow-up we observed for both fasting and $2 \mathrm{~h}$ glucose a stronger shift on the right side of the distribution curve in the control groups, with significantly more diabetes cases compared with the intervention groups.

The main strength of this study is that it is the first to report summary effects of lifestyle interventions on diabetes incidence in the high-risk South Asian population. Starting with a systematic review of the literature, we used individual participant data meta-analysis from all the six eligible RCTs among South Asians published prior to the inclusion date to produce precise summary effects overall and across subgroups [21]. The data were also analysed for the secondary outcomes: fasting and $2 \mathrm{~h}$ glucose and adiposity measures. Compared with meta-analyses based on aggregate data on a study level from published papers, individual participant data metaanalysis facilitates standardisation of analyses and increases the precision of estimates and the quality of subgroup analyses [21]. Further, assessing the quality of evidence according to GRADE, we systematically and transparently assessed all factors that could impact on our certainty in the effects estimates, including risk of bias in each study, heterogeneity, indirectness, imprecision and publication bias [32].

However, our review is limited by a relatively small number of trials, some with high loss to follow-up, and relatively little variation in settings. The included studies counted approximately 300 incident cases of diabetes, the majority (83\%) in the Indian studies. Thus, the power to detect subgroup differences and to further explore between-study heterogeneity for the adiposity measures was limited. Although we consider the evidence to be generally applicable to the target population, we cannot fully rule out potential indirectness due to differences between populations (country of origin or migrant status), interventions (type, content, intensity, mode of delivery, compliance) and settings (family- and community-based vs workplace).

Our meta-analysis included efficacy $[12,16]$ and more pragmatic trials $[15,17,18,26]$. The $35 \% \mathrm{RR}$ reduction in diabetes incidence resembles the RR reduction (39\%) reported in a standard meta-analysis of 19 efficacy and pragmatic diabetes prevention trials in individuals with impaired glucose tolerance or impaired fasting glucose after a mean of 2.6 years of active lifestyle intervention [13]. Although ten studies in the latter meta-analysis were conducted in Asia, the results for South Asians were not reported. Of note, the absolute benefit on diabetes prevention was higher in our study than in this meta-analysis (7.4\% vs $4.0 \%$, respectively) and so the NNT was lower (14 vs 25) [13]. Our findings compare well with the first efficacy studies, reporting $6.2-12 \%$ absolute risk reductions (NNT 16-8, respectively) $[8,9]$, but are somewhat stronger than those of a meta-analysis of translational studies to prevent diabetes in high-risk populations other than South Asians (RR reduction, 29\%; absolute risk reduction, 3\%) [14].

Meta-analysis of diabetes prevention trials indicated that combined dietary and physical activity interventions were more effective than either strategy type alone, but the number of studies was limited for single strategy studies [13]. More studies to date seem to indicate that the dietary interventions are more effective than physical activity interventions to reduce diabetes incidence in high-risk individuals. There is, however, evidence that interventions focused on increasing physical activity in individuals with impaired glucose tolerance are effective at inducing sustained reductions in $2 \mathrm{~h}$ glucose concentrations [44]. Although one of the studies with a physical activity intervention that was included in our study found effects on weight and waist [18], more studies comparing dietary and activity interventions for diabetes prevention are needed in other ethnic groups than of European origin.

Although we could not assess this, the intensity of the interventions in the long-term studies in our review was lower $[12,26]$ or comparable [16] with those of the first efficacy studies, which had stronger effects $[8,9]$. Even a low-cost community-based peer-support lifestyle intervention programme published too late to be included in our study found a $12 \%$ relative reduction in diabetes incidence in individuals with a high Indian diabetes risk score [33]. The smaller effect might be partly attributed to the selection of participants, with the majority having normal blood glucose levels or isolated impaired fasting glucose at baseline, as there are no interventions so far proven to reduce diabetes incidence in such individuals [33].

In contrast to our finding of an overall $35 \%$ reduction on diabetes incidence with a relative small mean $0.75 \mathrm{~kg}$ reduction in weight, studies in other populations found weight reduction to be the main driver of the effect $[8,9,13]$, i.e. $16 \%$ reduction in diabetes incidence for each $\mathrm{kg}$ of weight loss [45]. Of note, despite small changes in mean weight and waist circumference values, both for fasting glucose (primarily reflecting hepatic insulin resistance) and $2 \mathrm{~h}$ glucose (reflecting muscle insulin resistance), the intervention had a more profound effect on the right side of the distribution curve, indicating reduced insulin resistance [46]. Further, achievements of dietary and physical activity goals, even without weight loss, may improve $2 \mathrm{~h}$ glucose and reduce 


\section{Overall}

IDPP-1 (2006) [12]

PAMH (2012) [18]

Innva-Diab-DE-PLAN (2013) [17]

DHIAAN (2013) [15]

Indian SMS study (2013) [26]

PODOSA (2014) [16]

Total $\left(Q=0.225, p=1.00 ; I^{2}=0.0 \%\right)$

Men

IDPP-1 (2006) [12]

PAMH (2012) [18]

DHIAAN (2013) [15]

Indian SMS study (2013) [26]

PODOSA (2014) [16]

Total $\left(Q=0.824, p=0.94 ; I^{2}=0.0 \%\right)$

Women

IDPP-1 (2006) [12]

Innva-Diab-DE-PLAN (2013) [17]

DHIAAN (2013) [15]

PODOSA (2014) [16]

Total $\left(Q=1.203, p=0.75 ; I^{2}=0.0 \%\right)$

BMI $\geq 27.5 \mathrm{~kg} / \mathrm{m}^{2}$

IDPP-1 (2006) [12]

Innva-Diab-DE-PLAN (2013) [17]

DHIAAN (2013) [15]

Indian SMS study (2013) [26]

PODOSA (2014) [16]

Total $\left(Q=6.854, p=0.14 ; I^{2}=45.9 \%\right)$

BMI $<27.5 \mathrm{~kg} / \mathrm{m}^{2}$

IDPP-1 (2006) [12]

DHIAAN (2013) [15]

Indian SMS study (2013) [26]

PODOSA (2014) [16]

Total $\left(Q=1.923, p=0.59 ; I^{2}=9.5 \%\right)$

Age $>\mathbf{4 4}$ years

IDPP-1 (2006) [12]

DHIAAN (2013) [15]

Indian SMS study (2013) [26]

PODOSA (2014) [16]

Total $\left(Q=0.515, p=0.99 ; I^{2}=0.0 \%\right)$

Age $\leq \mathbf{4 4}$ years

IDPP-1 (2006) [12]

PAMH (2012) [18]

Innva-Diab-DE-PLAN (2013) [17]

DHIAAN (2013) [15]

Indian SMS study (2013) [26]

PODOSA (2014) [16]

Total $\left(Q=0.071, p=0.79 ; I^{2}=0.0 \%\right)$

Study duration: 5-7 months

PAMH (2012) [18]

Innva-Diab-DE-PLAN (2013) [17]

Total $\left(Q=0.143, p=0.99 ; I^{2}=0.0 \%\right)$

Study duration: $2-3$ years

IDPP-1 (2006) [12]

DHIAAN (2013) [15]

Indian SMS study (2013) [26]

PODOSA (2014) [16]

Total $\left(Q=0.153, p=0.98 ; I^{2}=0.0 \%\right)$

\section{European studies}

PAMH (2012) [18]

Innva-Diab-DE-PLAN (2013) [17]

DHIAAN (2013) [15]

PODOSA (2014) [16]

Total $\left(Q=0.011, p=0.94 ; I^{2}=0.0 \%\right)$

Indian studies

IDPP-1 (2006) [12]

Indian SMS study (2013) [26]

Total $\left(Q=0.011, p=0.94 ; I^{2}=0.0 \%\right)$

$\begin{array}{lc}47 / 120 & 74 / 133 \\ 1 / 85 & 1 / 54 \\ 1 / 92 & 1 / 88 \\ 7 / 280 & 10 / 250 \\ 50 / 271 & 73 / 266 \\ 12 / 85 & 17 / 86\end{array}$

$42 / 93$

$1 / 85$

$4 / 136$

$50 / 271$

$5 / 39$

$59 / 100$

$1 / 54$

$3 / 128$

$73 / 266$

$6 / 39$

$5 / 27$

$1 / 92$

$3 / 147$

$7 / 44$

$15 / 33$

$1 / 88$

$7 / 124$

$11 / 46$

$5 / 127$

$17 / 64$

$8 / 55$

$23 / 35$

$1 / 54$

$6 / 123$

$16 / 67$

$13 / 58$

41/94

2/156

$33 / 207$

$4 / 26$

$51 / 98$

$4 / 130$

$57 / 199$

$4 / 24$

29/71

$4 / 153$

$28 / 146$

9/68

40/76

$6 / 121$

$6 / 121$
$42 / 154$

$12 / 64$

$18 / 49$

$1 / 73$

$1 / 64$

$3 / 130$

22/125

$3 / 13$

$34 / 57$

$1 / 38$

$1 / 58$

$4 / 132$

$31 / 112$

$5 / 18$

$1 / 85$

$1 / 92$

$1 / 54$

$1 / 88$

$1 / 92$

$7 / 280$

$50 / 271$

$12 / 85$

$1 / 88$

$10 / 250$

$73 / 266$

$17 / 86$

$1 / 85$

$1 / 92$

$7 / 280$

$12 / 85$

$1 / 54$

$1 / 88$

$10 / 250$

$17 / 86$

$47 / 120$

$50 / 271$

$74 / 133$

$73 / 266$
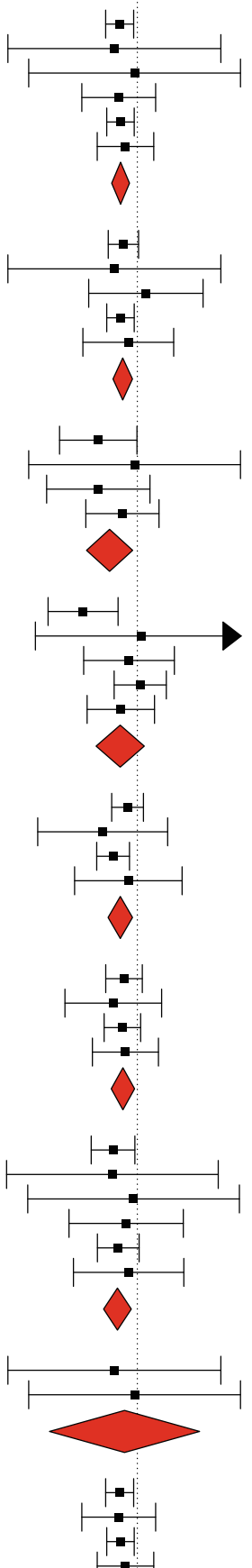

$\rightarrow$

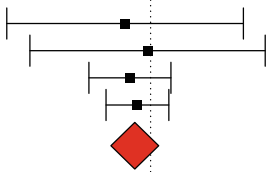

$0.63[0.44,0.91]$

$0.55[0.03,8.91]$

$0.93[0.06,14.90]$

$0.61[0.23,1.62]$

$0.64[0.45,0.92]$

$0.73[0.35,1.54]$

$0.65[0.51,0.81]$

$0.69[0.47,1.03]$

$0.55[0.03,8.91]$

$1.25[0.28,5.59]$

$0.64[0.45,0.92]$

$0.79[0.24,2.59]$

$0.68[0.53,0.88]$

$0.36[0.13,0.99]$

$0.93[0.06,14.90]$

$0.36[0.09,1.39]$

$0.68[0.26,1.76]$

$0.48[0.26,0.88]$

$0.24[0.10,0.60]$

$1.11[0.07,17.74]$

$0.80[0.25,2.64]$

$1.08[0.54,2.14]$

$0.65[0.27,1.57]$

$0.64[0.34,1.20]$

$0.77[0.51,1.17]$

$0.40[0.07,2.21]$

$0.53[0.35,0.81]$

$0.79[0.19,3.23]$

0.64 [0.46, 0.88]

$0.71[0.44,1.14]$

$0.53[0.15,1.89]$

$0.68[0.42,1.09]$

$0.73[0.31,1.74]$

$0.69[0.50,0.93]$

$0.53[0.30,0.94]$ $0.52[0.03,8.32]$

$0.91[0.06,14.49]$

$0.75[0.17,3.33]$

$0.61[0.35,1.05]$

$0.80[0.19,3.38]$

$0.59[0.41,0.85]$

$0.55[0.03,8.91]$ $0.93[0.06,14.90]$

$0.72[0.10,5.11]$

$0.63[0.44,0.91]$ $0.61[0.23,1.62]$ $0.64[0.45,0.92]$ $0.73[0.35,1.54]$

$0.64[0.51,0.81]$

$0.55[0.03,8.91]$ $0.93[0.06,14.90]$ $0.61[0.23,1.62]$

$0.73[0.35,1.54]$

$0.69[0.39,1.21]$

$0.63[0.44,0.91]$ $0.64[0.45,0.92]$

$0.64[0.49,0.82]$ 
Fig. 1 Forest plot of HRs for diabetes in lifestyle intervention vs control groups, overall and for subgroups. IDPP-1, Indian Diabetes Prevention Programme; Innva-Diab-DE-PLAN, the InnvaDiab (Immigrant Diabetes) study, part of the European Union project DE-PLAN (Diabetes in Europe - Prevention using Lifestyle, Physical Activity and Nutritional Intervention); PAMH, Physical Activity and Minority Health; SMS, short message service

diabetes incidence [8], as found in the Indian studies in our review $[12,26]$, where a reduction in portion size, consumption of carbohydrates and oil intake was related to a lower diabetes incidence even without weight loss [39]. Although trials among South Asians are few, there are indications that improvements in the quality of the diet (more complex carbohydrates and monounsaturated and polyunsaturated fatty acids) might improve blood glucose, serum insulin, lipids, inflammatory markers and hepatic fat [5].

Although only $33 \%$ of participants were women in our meta-analysis, as previous studies were underpowered to study potential sex differences, an important new finding was that their diabetes incidence was significantly reduced, despite a slightly smaller non-significant effect estimate for $2 \mathrm{~h}$ glucose, weight and waist circumference than in men. Furthermore, the larger effect on weight, with a concomitant non-significant effect estimate for waist circumference, in the European compared with the Indian studies in our metaanalysis is noteworthy. This may reflect different phenotypes, as it would be easier to achieve a larger reduction in weight measures among South Asians residing in Europe, who had higher BMIs, but differences in the diet and/or physical activity level at baseline or induced by the intervention may also be involved. Furthermore, perceptions of weight and health are changing across generations and differ by region [47]. South Asians living in Europe, particularly women, may be more sensitised to the importance of weight loss than those living in their country of origin [48]. Others report that sex did not influence the effects of lifestyle interventions [13]. Lifestyle trials aimed at weight loss which explored sex differences in anthropometric outcomes mostly report stronger effects in men, but actual sex differences were small [49]. Thus, there is little evidence yet to indicate that men and women should generally adopt different weight loss strategies.

The results of our meta-analysis, that lifestyle interventions had comparable and clinically important effects on diabetes incidence across different regions, is important for policymakers and clinicians. The studies outside India in our meta-analysis, conducted mostly among first-generation South Asian migrants in Europe, had suggested only a modest effect. The consistency of effects across subgroups of the South Asian population at risk of diabetes has made us rethink, contrary to our a priori beliefs based on the outcomes of single studies, that benefits may actually be achieved by lifestyle modification interventions, not only under ideal conditions, but also in real-life settings $[15,17,18,26]$. Cultural adaptations to mode of delivery may be necessary across contexts as cultural adaptations likely promote the effectiveness of interventions among specific ethnic populations [50, 51], although evidence of the effects of cultural targeting on diabetes prevention outside India is still scarce [52]. Interestingly, one study using a culturally targeted physical activity intervention (floorball/field hockey) for men provided strong results for all secondary outcomes, although it was limited by its small size and short duration [18].

There are, however, several unanswered questions that should be addressed. First, lifestyle intervention studies have used generic recommendations (i.e. based on those for the local majority population) [27], while different targets for dietary and physical activity recommendations may be necessary [27, 53]. Further work is required on the mechanisms by which these interventions are having their effect, including the role and type of physical activity, diet quality, specific dietary components,

Table 3 Overall effects of lifestyle interventions (mean difference) on continuous outcomes

\begin{tabular}{|c|c|c|c|c|c|c|c|}
\hline \multirow[t]{2}{*}{ Outcome } & \multirow[t]{2}{*}{ No. of studies } & \multirow[t]{2}{*}{ No. of participants } & \multicolumn{2}{|c|}{ Mean (SD) kg } & \multirow[t]{2}{*}{ Summary adjusted $\mathrm{MD}^{\mathrm{a}}(95 \% \mathrm{CI})$} & \multirow[t]{2}{*}{$p$ value ${ }^{\mathrm{b}}$} & \multirow[t]{2}{*}{$I^{2}(\%)$} \\
\hline & & & Intervention & Control & & & \\
\hline Fasting glucose (mmol/l) & 6 & 1411 & $5.5(0.90)$ & $5.6(1.15)$ & $-0.03(-0.10,0.04)$ & 0.37 & 0 \\
\hline $2 \mathrm{~h}$ glucose $(\mathrm{mmol} / \mathrm{l})$ & 6 & 1428 & $7.4(2.44)$ & $8.1(3.09)$ & $-0.34(-0.62,-0.07)$ & 0.02 & 50 \\
\hline Weight $(\mathrm{kg})$ & 6 & 1479 & $73.7(13.08)$ & $74.1(12.44)$ & $-0.75(-1.34,-0.17)$ & 0.01 & 71 \\
\hline Waist (cm) & 6 & 1462 & $93.7(10.18)$ & $94.5(9.73)$ & $-1.16(-2.16,-0.16)$ & 0.02 & 75 \\
\hline
\end{tabular}

Data are pooled with last estimate

${ }^{a}$ Mean difference based on last estimate (PODOSA and IDPP-1 = 3 years, DHIAAN and Indian SMS study = 2 years, Innva-Diab-DE-PLAN = 7 months and PAMH $=5$ months), adjusted for age and baseline values for outcome variable

${ }^{\mathrm{b}} p$ values for mean difference. Analyses are based on participants with at least two measurements. Two studies (DHIAAN, Innva-Diab-DE-PLAN) had > $20 \%$ missing data at follow-up (similar for intervention and control groups)

IDPP-1, Indian Diabetes Prevention Programme; Innva-Diab-DE-PLAN, the InnvaDiab (Immigrant Diabetes) study, part of the European Union project DE-PLAN (Diabetes in Europe - Prevention using Lifestyle, Physical Activity and Nutritional Intervention); MD, mean difference; No., number; PAMH, Physical Activity and Minority Health; SMS, short message service 
cooking practices and timing of meals [54]. Further, we predict that larger effects on diabetes risk in South Asians might be achieved in studies aiming at larger weight reductions [55, 56] and with more intense dietary and physical activity changes [53]. Reach, retention and long-term sustainability may be enhanced through improvements in targeting and delivery, and benefits in the longer term (beyond 2-3 years) should be further investigated. It is hoped that ongoing studies will be complemented with new high-quality trials addressing the issues outlined above. Future systematic reviews should be extended to explore effects by mode of delivery, intensity and contextual factors. In addition to high-risk strategies, there are strong recommendations for population-based strategies as part of national public health policies [54].

In conclusion, pending deeper understanding of the causation of diabetes in South Asians and the development of new kinds of intervention, this individual participant data meta-analysis of lifestyle modification interventions in South Asian populations at high risk of diabetes provides evidence of a clinically important $35 \%$ relative reduction in diabetes incidence, with an NNT of 14 to prevent one case of diabetes over a mean of 2 years. Given the substantial and growing burden of diabetes, particularly in South Asian origin populations, this meta-analysis provides support for ongoing strategies underpinned by weight loss, dietary change and increased physical activity to prevent diabetes. Future work should aim to understand the mechanisms by which these effects occur, evaluate cost effectiveness and develop more effective interventions.

Acknowledgements We thank F.S. van Etten-Jamaludin (clinical librarian, Amsterdam UMC, location Academic Medical Center [AMC]) for her help with the development of the search strategy for the different databases. We also thank M. Nicolaou (AMC) and S. Quereshi (Norwegian Centre for Migration and Minority Health, Norwegian Institute of Public Health, Oslo, Norway) for their contribution to the systematic review data collection on which the present study builds [27].

Some of the data were presented as an abstract at the 54th Annual Meeting of the EASD in Berlin, 2018 (S160, Abstract 317).

Data availability Data from the individual studies were obtained through the principal investigators of the individual studies for the purpose of the current analysis only, after formal collaboration and data transfer agreements were signed. These agreements restrict the further dissemination of data to third parties. The team of the current study may be contacted with further questions about these procedures. Additionally, researchers interested in the data of the individual studies may contact the principal investigators of the respective trials with their data requests.

Funding The work was sponsored by the Health Program 2014-2020 from the European Union, grant number 664609 HPPJ-2014, set up to improve the prevention of diabetes in South Asians, and by the authors' institutions: University of Oslo, Oslo, Norway (AKJ, IM, POV); Oslo Metropolitan University, Oslo, Norway (IB, KRR); and Amsterdam UMC, location AMC, University of Amsterdam, the Netherlands (MM, $\mathrm{EB}, \mathrm{KS}, \mathrm{IGMvV})$. The funders had no role in study design, data collection, analysis, data interpretation or writing of the paper. No pharmaceutical companies or representatives have been involved.
Duality of interest The authors declare that there is no duality of interest associated with this manuscript. Some authors contributed to studies that were included in this systematic review. JMRG, NS, RSB, AD and AS were co-authors on the Prevention of Diabetes and Obesity in South Asians (PODOSA) trial but were not involved in its evaluation in the present study. IGMvV and KS contributed to the Diabetes Intervention Study in Hindustani Surinamese (DHIAAN), and were therefore not involved in the quality assessment in the present study. Similarly, no other co-authors who contributed their data (AR, MK, EA) were involved in the quality assessment in the present study.

Contribution statement The present study builds on a previous systematic review in which the corresponding author was involved. RSB, KS and IGMvV conceived and initiated the meta-analysis and AKJ and IGMvV designed the current study. All authors (except RSB) were involved in designing, and agreed on, the protocol for analysis. MM and IGMvV screened abstracts and later full-text studies for eligibility. $\mathrm{MM}, \mathrm{IGMvV}$ and CAC-M assessed the quality of eligible studies. Data were acquired by IB and IM. IGMvV, KS, AR, MK, EA, AD, GC and AS contributed data to the study and verified preliminary results for their respective studies. IB, IM and KRR analysed the data and, together with AKJ, POV and IGMvV, interpreted the initial results. AKJ and IGMvV drafted the manuscript, with input from MM, IB, IM and POV. All authors contributed to the interpretation of data, critically revised the manuscript and approved the last version for publication. IB, IM, KRR and AKJ had full access to all the data in the study. AKJ is responsible for the integrity of the work as a whole.

\section{References}

1. Sattar N, Gill JMR (2015) Type 2 diabetes in migrant south Asians: mechanisms, mitigation, and management. Lancet Diabetes Endocrinol 3(12):1004-1016. https://doi.org/10.1016/S22138587(15)00326-5

2. Jenum AK, Diep LM, Holmboe-Ottesen G, Holme IM, Kumar BN, Birkeland KI (2012) Diabetes susceptibility in ethnic minority groups from Turkey, Vietnam, Sri Lanka and Pakistan compared with Norwegians - the association with adiposity is strongest for ethnic minority women. BMC Public Health 12(1):150. https://doi. org/10.1186/1471-2458-12-150

3. Gholap N, Davies M, Patel K, Sattar N, Khunti K (2011) Type 2 diabetes and cardiovascular disease in South Asians. Prim Care Diabetes 5(1):45-56. https://doi.org/10.1016/j.pcd.2010.08.002

4. Raymond NT, Varadhan L, Reynold DR et al (2009) Higher prevalence of retinopathy in diabetic patients of South Asian ethnicity compared with white Europeans in the community: a crosssectional study. Diabetes Care 32(3):410-415. https://doi.org/10. 2337/dc08-1422

5. Gulati S, Misra A (2017) Abdominal obesity and type 2 diabetes in Asian Indians: dietary strategies including edible oils, cooking practices and sugar intake. Eur J Clin Nutr 71(7):850-857. https://doi. org/10.1038/ejen.2017.92

6. Krishnaveni GV, Yajnik CS (2017) Developmental origins of diabetes-an Indian perspective. Eur J Clin Nutr 71(7):865-869. https://doi.org/10.1038/ejen.2017.87

7. Ikram UZ, Kunst AE, Lamkaddem M, Stronks K (2014) The disease burden across different ethnic groups in Amsterdam, the Netherlands, 2011-2030. Eur J Pub Health 24(4):600-605. https:// doi.org/10.1093/eurpub/ckt136

8. Tuomilehto J, Lindström J, Eriksson JG et al (2001) Prevention of type 2 diabetes mellitus by changes in lifestyle among subjects with impaired glucose tolerance. N Engl J Med 344(18):1343-1350. https://doi.org/10.1056/NEJM200105033441801 
9. Knowler WC, Barrett-Connor E, Fowler SE et al (2002) Reduction in the incidence of type 2 diabetes with lifestyle intervention or metformin. N Engl J Med 346(6):393-403. https://doi.org/10. 1056/NEJMoa012512

10. Pan XR, Li GW, Hu YH et al (1997) Effects of diet and exercise in preventing NIDDM in people with impaired glucose tolerance: the Da Qing IGT and diabetes study. Diabetes Care 20(4):537-544. https://doi.org/10.2337/diacare.20.4.537

11. Kosaka K, Noda M, Kuzuya T (2005) Prevention of type 2 diabetes by lifestyle intervention: a Japanese trial in IGT males. Diabetes Res Clin Pract 67(2):152-162. https://doi.org/10.1016/j.diabres.2004. 06.010

12. Ramachandran A, Snehalatha C, Mary S et al (2006) The Indian Diabetes Prevention Programme shows that lifestyle modification and metformin prevent type 2 diabetes in Asian Indian subjects with impaired glucose tolerance (IDPP-1). Diabetologia 49(2):289-297. https://doi.org/10.1007/s00125-005-0097-z

13. Haw JS, Galaviz KI, Straus AN et al (2017) Long-term sustainability of diabetes prevention approaches: a systematic review and meta-analysis of randomized clinical trials. JAMA Intern Med 177(12):1808-1817. https://doi.org/10.1001/jamainternmed.2017. 6040

14. Galaviz KI, Weber MB, Straus A, Haw JS, Narayan KMV, Ali MK (2018) Global diabetes prevention interventions: a systematic review and network meta-analysis of the real-world impact on incidence, weight, and glucose. Diabetes Care 41(7):1526-1534. https://doi.org/10.2337/dc17-2222

15. Admiraal WM, Vlaar EM, Nierkens V et al (2013) Intensive lifestyle intervention in general practice to prevent type 2 diabetes among 18 to 60 -year-old South Asians: 1-year effects on the weight status and metabolic profile of participants in a randomized controlled trial. PLoS One 8(7):e68605. https://doi.org/10.1371/ journal.pone. 0068605

16. Bhopal RS, Douglas A, Wallia S et al (2014) Effect of a lifestyle intervention on weight change in south Asian individuals in the UK at high risk of type 2 diabetes: a family-cluster randomised controlled trial. Lancet Diabetes Endocrinol 2(3):218-227. https://doi. org/10.1016/S2213-8587(13)70204-3

17. Telle-Hjellset V, Kjollesdal MKR, Bjorge B et al (2013) The InnvaDiab-DE-PLAN study: a randomised controlled trial with a culturally adapted education programme improved the risk profile for type 2 diabetes in Pakistani immigrant women. Br J Nutr 109(3):529-538. https://doi.org/10.1017/S000711451200133X

18. Andersen E, Burton NW, Anderssen SA (2012) Physical activity levels six months after a randomised controlled physical activity intervention for Pakistani immigrant men living in Norway. Int J Behav Nutr Phys Act 9(1):47. https://doi.org/10.1186/1479-5868-9-47

19. Brown T, Smith S, Bhopal R, Kasim A, Summerbell C (2015) Diet and physical activity interventions to prevent or treat obesity in South Asian children and adults: a systematic review and metaanalysis. Int J Environ Res Public Health 12(1):566-594. https:// doi.org/10.3390/ijerph120100566

20. Chapman J, Qureshi N, Kai J (2013) Effectiveness of physical activity and dietary interventions in south Asian populations: a systematic review. Br J Gen Pract 63(607):e104-e114. https://doi. org/10.3399/bjgp13X663064

21. Riley RD, Lambert PC, Abo-Zaid G (2010) Meta-analysis of individual participant data: rationale, conduct, and reporting. BMJ 340: c221. https://doi.org/10.1136/bmj.c221

22. Shamseer L, Moher D, Clarke M et al (2015) Preferred reporting items for systematic review and meta-analysis protocols (PRISMAP) 2015: elaboration and explanation. BMJ 349:g7647. https://doi. org/10.1136/bmj.g7647

23. Thomas H (2003) Quality assessment tool for quantitative studies: effective public health practice project. McMaster University, Hamilton, ON, Canada
24. Chowdhury TA, Hitman GA (2007) Type 2 diabetes in people of South Asian origin: potential strategies for prevention. Br J Diabetes Vasc Dis 7(6):279-282. https://doi.org/10.1177/ 14746514070070060401

25. Ramachandran A, Snehalatha C, Samith Shetty A, Nanditha A (2013) Primary prevention of Type 2 diabetes in South Asianschallenges and the way forward. Diabet Med 30(1):26-34. https:// doi.org/10.1111/j.1464-5491.2012.03753.x

26. Ramachandran A, Snehalatha C, Ram J et al (2013) Effectiveness of mobile phone messaging in prevention of type 2 diabetes by lifestyle modification in men in India: a prospective, parallel-group, randomised controlled trial. Lancet Diabetes Endocrinol 1(3):191198. https://doi.org/10.1016/S2213-8587(13)70067-6

27. Muilwijk M, Nicolaou M, Qureshi SA et al (2018) Dietary and physical activity recommendations to prevent type 2 diabetes in South Asian adults: a systematic review. PLoS One 13(7): e0200681. https://doi.org/10.1371/journal.pone.0200681

28. WHO Expert Consultation (2004) Appropriate body-mass index for Asian populations and its implications for policy and intervention strategies. Lancet 363(9403):157-163

29. Koopman L, van der Heijden GJ, Grobbee DE, Rovers MM (2008) Comparison of methods of handling missing data in individual patient data meta-analyses: an empirical example on antibiotics in children with acute otitis media. Am J Epidemiol 167(5):540-545. https://doi.org/10.1093/aje/kwm341

30. Carpenter JR, Goldstein H, Michael G, Kenward MG (2011) REALCOM-IMPUTE software for multilevel multiple imputation with mixed response types. J Stat Softw 45(5):1-14

31. Royston P, White IR (2011) Multiple imputation by chained equations (MICE): implementation in Stata. J Stat Softw 45(5):1-20

32. Guyatt GH, Oxman AD, Vist GE et al (2008) GRADE: an emerging consensus on rating quality of evidence and strength of recommendations. BMJ 336(7650):924-926. https://doi.org/10.1136/bmj. 39489.470347.AD

33. Thankappan KR, Sathish T, Tapp RJ et al (2018) A peer-support lifestyle intervention for preventing type 2 diabetes in India: a cluster-randomized controlled trial of the Kerala Diabetes Prevention Program. PLoS Med 15(6):e1002575. https://doi.org/ 10.1371/journal.pmed.1002575

34. Mayer-Davis EJ, Sparks KC, Hirst K et al (2004) Dietary intake in the diabetes prevention program cohort: baseline and 1-year post randomization. Ann Epidemiol 14(10):763-772. https://doi.org/10. 1016/j.annepidem.2004.02.004

35. Snehalatha C, Mary S, Joshi VV, Ramachandran A (2008) Beneficial effects of strategies for primary prevention of diabetes on cardiovascular risk factors: results of the Indian Diabetes Prevention Programme. Diab Vasc Dis Res 5(1):25-29. https:// doi.org/10.3132/dvdr.2008.005

36. Snehalatha C, Mary S, Selvam S et al (2009) Changes in insulin secretion and insulin sensitivity in relation to the glycemic outcomes in subjects with impaired glucose tolerance in the Indian Diabetes Prevention Programme-1 (IDPP-1). Diabetes Care 32(10):1796-1801. https://doi.org/10.2337/dc09-0676

37. Ramachandran A, Arun N, Shetty AS, Snehalatha C (2010) Efficacy of primary prevention interventions when fasting and postglucose dysglycemia coexist: analysis of the Indian Diabetes Prevention Programmes (IDPP-1 and IDPP-2). Diabetes Care 33(10):2164-2168. https://doi.org/10.2337/dc09-1150

38. Patel JV, Hughes EA, Lip GY, Gill PS (2011) Diabetes Health, Residence \& Metabolism in Asians: the DHRMA study, research into foods from the Indian subcontinent - a blinded, randomised, placebo controlled trial. BMC Cardiovasc Disord 11(1):70. https:// doi.org/10.1186/1471-2261-11-70

39. Ram J, Selvam S, Snehalatha C et al (2014) Improvement in diet habits, independent of physical activity helps to reduce incident diabetes among prediabetic Asian Indian men. Diabetes Res Clin 
Pract 106(3):491-495. https://doi.org/10.1016/j.diabres.2014.09. 043

40. Weber MB, Ranjani H, Staimez LR et al (2016) The stepwise approach to diabetes prevention: results from the D-CLIP randomized controlled trial. Diabetes Care 39(10):1760-1767. https://doi.org/ $10.2337 / \mathrm{dc} 16-1241$

41. Willis A, Roshan M, Patel N et al (2016) A community faith centre based screening and educational intervention to reduce the risk of type 2 diabetes: a feasibility study. Diabetes Res Clin Pract 120:7380. https://doi.org/10.1016/j.diabres.2016.07.025

42. Patel RM, Misra R, Raj S, Balasubramanyam A (2017) Effectiveness of a group-based culturally tailored lifestyle intervention program on changes in risk factors for type 2 diabetes among Asian Indians in the United States. J Diabetes Res 2017:2751980

43. Wijesuriya M, Fountoulakis N, Guess N et al (2017) A pragmatic lifestyle modification programme reduces the incidence of predictors of cardio-metabolic disease and dysglycaemia in a young healthy urban South Asian population: a randomised controlled trial. BMC Med 15(1):146. https://doi.org/10.1186/s12916-0170905-6

44. Yates T, Davies M, Gorely T, Bull F, Khunti K (2009) Effectiveness of a pragmatic education programme aimed at promoting walking activity in individuals with impaired glucose tolerance: a randomized controlled trial. Diabetes Care 32(8):1404-1410. https://doi. org/10.2337/dc09-0130

45. Hamman RF, Wing RR, Edelstein SL et al (2006) Effect of weight loss with lifestyle intervention on risk of diabetes. Diabetes Care 29(9):2102-2107. https://doi.org/10.2337/dc06-0560

46. Engberg S, Glumer C, Witte DR, Jorgensen T, Borch-Johnsen K (2010) Differential relationship between physical activity and progression to diabetes by glucose tolerance status: the Inter99 Study. Diabetologia. 53(1):70-78. https://doi.org/10.1007/s00125-009$1587-1$

47. Patel S, Bhopal R, Unwin N, White M, Alberti KG, Yallop J (2001) Mismatch between perceived and actual overweight in diabetic and non-diabetic populations: a comparative study of South Asian and European women. J Epidemiol Community Health 55(5):332-333. https://doi.org/10.1136/jech.55.5.332

48. Hussain A, Bjorge B, Hjellset VT, Holmboe-Ottesen G, Wandel M (2010) Body size perceptions among Pakistani women in Norway participating in a controlled trial to prevent deterioration of glucose tolerance. Ethn Health 15(3):237-251. https://doi.org/10.1080/ 13557851003668148
49. Williams RL, Wood LG, Collins CE, Callister R (2015) Effectiveness of weight loss interventions - is there a difference between men and women: a systematic review. Obes Rev 16(2): 171-186. https://doi.org/10.1111/obr.12241

50. Davidson EM, Liu JJ, Bhopal R et al (2013) Behavior change interventions to improve the health of racial and ethnic minority populations: a tool kit of adaptation approaches. The Milbank Quarterly 91(4):811-851. https://doi.org/10.1111/1468-0009. 12034

51. Kreuter MW, Lukwago SN, Bucholtz RD, Clark EM, SandersThompson V (2003) Achieving cultural appropriateness in health promotion programs: targeted and tailored approaches. Health Educ Behav 30(2):133-146. https://doi.org/10.1177/1090198102251021

52. Nierkens V, Hartman MA, Nicolaou M et al (2013) Effectiveness of cultural adaptations of interventions aimed at smoking cessation, diet, and/or physical activity in ethnic minorities. a systematic review. PLoS One 8(10):e73373. https://doi.org/10.1371/journal. pone. 0073373

53. Iliodromiti S, Ghouri N, Celis-Morales CA, Sattar N, Lumsden MA, Gill JM (2016) Should physical activity recommendations for South Asian adults be ethnicity-specific? Evidence from a cross-sectional study of South Asian and White European men and women. PLoS One 11(8):e0160024. https://doi.org/10.1371/ journal.pone.0160024

54. Bhopal RS (2019) Epidemic of cardiovascular disease and diabetes. Explaining the phenomenon in South Asians worldwide. Oxford University Press, Oxford. https://doi.org/10.1093/med/ 9780198833246.001.0001

55. Lean ME, Leslie WS, Barnes AC et al (2018) Primary care-led weight management for remission of type 2 diabetes (DiRECT): an open-label, cluster-randomised trial. Lancet 391(10120):541551. https://doi.org/10.1016/S0140-6736(17)33102-1

56. Huttunen-Lenz M, Hansen S, Christensen P et al (2018) PREVIEW study-influence of a behavior modification intervention (PREMIT) in over 2300 people with pre-diabetes: intention, self-efficacy and outcome expectancies during the early phase of a lifestyle intervention. Psychol Res Behav Manag 11:383-394. https://doi.org/10. 2147/PRBM.S160355

Publisher's note Springer Nature remains neutral with regard to jurisdictional claims in published maps and institutional affiliations. 